\title{
Treatment with istradefylline for postural abnormalities in Parkinson's disease
}

\author{
Angela B. Deutschländer \\ Department of Neurology, Mayo Clinic, 4500 San Pablo Rd S, Jacksonville, FL 32225, U.S.A.
}

\begin{abstract}
Introduction. In the current edition, Fujioka and colleagues report on four Japanese patients with Parkinson disease (PD) and severe postural abnormalities treated with istradefylline (adenosine A2A receptor antagonist); further, dopamine agonists were withdrawn. Three patients experienced significant improvements of postural abnormalities.

Clinical reflections. Postural abnormalities in PD include camptocormia, antecollis, lateral trunk flexion, and scoliosis. They may be very pronounced and significantly reduce quality of life. The therapy of postural deformities in PD is currently disappointing. Clinical implications. Effective therapeutic strategies for postural deformities in PD are an unmet need. Larger clinical trials investigating novel approaches including istradefylline are warranted.
\end{abstract}

(Neurol Neurochir Pol 2019; 53 (4): 239-241)

Istradefylline is a selective adenosine $\mathrm{A} 2 \mathrm{~A}$ receptor (A2AR) antagonist, which is approved for treatment in patients with Parkinson's disease (PD) in Japan. It has been submitted for FDA approval for use as adjunctive treatment to L-dopa in patients with PD experiencing OFF episodes. Fujioka and colleagues describe four Japanese patients with PD and postural abnormalities (antecollis, "Pisa syndrome", and camptocormia), in whom treatment with istradefylline was initiated [1]. Further, their dopaminergic medication was discontinued simultaneously or up to two months before starting istradefylline. Three patients had well-preserved paraspinal muscle volume and showed moderate to very good improvement of postural abnormalities. The fourth patient had moderate paraspinal muscle atrophy and experienced no improvement of her antecollis. In conclusion, istradefylline may be a novel therapeutic strategy for postural abnormalities in PD.

Postural abnormalities in Parkinson's disease include camptocormia, antecollis, lateral trunk flexion (LTF, "Pisa syndrome") and scoliosis. The term "camptocormia" was coined in 1915 [2]. The syndrome was initially described by Brodie in 1937 as "hysterical bent back" [3]. Since consensus criteria for camptocormia are lacking, thoracolumbar flexion angles of $\geq$ 45 degree are generally chosen for the diagnosis. Accordingly, the estimated prevalence of camptocormia varies (3-18\%). Camptocormia and LTF are generally aggravated by walking or exercising and alleviated by lying supine, standing against a wall, using walking support, or in few instances by wearing a low-slung backpack, thus exhibiting features of dystonia, including a "sensory trick" $[4,5]$. Scoliosis, however, remains fixed, and its diagnosis requires imaging of the spine [5]. Postural deformities are more frequent in female patients with PD, who are older, have more advanced disease, and they appear to occur more frequently in Asia. A recent study reported that 9/18 patients with $\mathrm{PD}$ and postural deformities ( $\geq 1$ point on the UPDRS III item "posture"; all patients were examined in the ON state) showed improved posture during istradefylline treatment. The patients who responded had higher Hoehn and Yahr stages [6]. A positive response to istradefylline was further observed in a case series, in which camptocormia was mostly OFF-state associated [7].

The etiology of postural abnormalities in PD remains poorly understood $[4,5,8]$. Camptocormia in PD was reported to

Address for correspondence: Angela B. Deutschländer M.D., Ph.D., Department of Neurology, Mayo Clinic Florida, 4500 San Pablo Rd S., Jacksonville, FL 32225, U.S.A. e-mail: Deutschlander.Angela@mayo.edu 
worsen in the OFF state and thus suggested to arise due to rigidity. Further etiologies of camptocormia include primary and secondary myopathy, proprioceptive dysregulation, dystonia, inflammation/myositis as well as functional and medicationinduced disorders. Additionally, an impaired perception of verticality is associated with postural abnormalities in $\mathrm{PD}[4,5,8]$. Medications that may cause postural abnormalities include dopamine agonists, anticholinergics, and amantadine. The notion that dopamine agonist may induce reversible postural abnormalities is currently based on case reports, which mostly stem from Japan [9-15]. L-dopa and dopaminergic agents may worsen, improve or not alter postural abnormalities in PD [4, 16]. Finally, a consistent myopathic lesion pattern was seen in paraspinal muscle biopsies of patients with camptocormia and PD [17].

Transgenic mice with the DYT1 mutation showed reduced dopamine D2 receptor (D2R) protein and reduced ability of D2Rs to activate their cognate Go/i proteins. Resulting synaptic plasticity impairment was fully restored by pharmacological blockade of A2ARs [18]. Thus, istradefylline may improve dystonic components of postural abnormalities. Further, istradefylline may reduce rigidity by inhibiting basal ganglia output through an indirect pathway; it may reduce the cholinergic output of the pedunculopontine nucleus (PPN) [19-20]. The PPN is assumed to reduce muscle tone via $\alpha$ - and $\gamma$-motoneurons. Blockage of GABAergic nucleus accumbens output to brainstem centers including the mesencephalic locomotor region has also been postulated [21].

Current treatment of postural abnormalities in PD is challenging. Regarding injections with botulinum toxin i.m., differentiation between upper and lower camptocormia has been proposed [4], since different muscles appear involved in these two distinct types of camptocormia. In most studies, the rectus abdominis and the iliopsoas muscles were injected [4]. Some success was achieved using $1 \%$ lidocaine [22]. Other pharmacological options include trihexiphenidyl, baclofen, amantadine, biperiden, tetrabenazine, clonazepam and bromazepam; efficacy, however, is considered disappointing $[4,5,8]$. L-dopa improved posture in about $20 \%$ of patients with PD [16]. One case series found that i.v. L-dopa infusions improved camptocormia and antecollis in PD, especially when abnormal postures were OFF-state associated [23]. Surgical approaches include deep brain stimulation (of about 80 patients, about $60 \%$ showed improvement of posture); orthopedic spinal correction, and unilateral pallidotomy [8]. Lastly, physiotherapy can achieve improvements.

Several randomized placebo-controlled studies including multicenter phase 3 studies (https://clinicaltrials.gov/), meta-analyses, and reviews [24-27] evaluating the efficacy of istradefylline in PD are available. Overall, istradefylline is considered to decrease OFF time duration and improve motor features in PD. However, not all studies reported positive results [28]. Several studies found only moderate improvements. E.g., the KW-6002-US-2018 study showed only modest changes in UPDRS III scores, and istradefylline did not significantly impact OFF time duration [29]. One meta-analysis found positive results only for istradefylline $40 \mathrm{mg} / \mathrm{d}$ but not $20 \mathrm{mg} / \mathrm{d}$ [25]. In a recent post-marketing surveillance study, UPDRS III scores decreased from 33.7 to 29.2 after treatment over one year; OFF time duration was reduced in only $38.2 \%$ of the patients [30]. While adverse effects like dyskinesia or nausea do occur, istradefylline is generally considered well tolerated.

In summary, postural abnormalities in PD can be very severe, and therapeutic strategies are an unmet need. Thus, larger clinical studies on therapeutic approaches are warranted, both regarding surgical procedures and pharmacological approaches including novel agents like A2AR antagonists.

\section{Conflict of interest: none}

\section{References}

1. Fujioka S, Yoshida R, Nose K, et al. A new therapeutic strategy with istradefylline for postural deformities in Parkinson's disease. Neurol Neurochir Pol. 2019; 53 (4): 291-295, doi: 10.5603/PJNNS. a2019.0036, indexed in Pubmed: 31441493.

2. Wartenberg R. Camptocormia. Arch Neurol Psychiatry. 1946; 56: 327 , indexed in Pubmed: 20999834.

3. Rosen JC, Frymoyer JW. A review of camptocormia and an unusual case in the female. Spine (Phila Pa 1976). 1985; 10(4): 325-327, doi: 10.1097/00007632-198505000-00006, indexed in Pubmed: 2931830.

4. Srivanitchapoom P, Hallett M. Camptocormia in Parkinson's disease: definition, epidemiology, pathogenesis and treatment modalities. J Neurol Neurosurg Psychiatry. 2016; 87(1): 75-85, doi: 10.1136/ jnnp-2014-310049, indexed in Pubmed: 25896683.

5. Doherty KM, van de Warrenburg BP, Peralta MC, et al. Postural deformities in Parkinson's disease. Lancet Neurol. 2011; 10(6): 538-549, doi: 10.1016/S1474-4422(11)70067-9, indexed in Pubmed: 21514890.

6. Suzuki K, Miyamoto T, Miyamoto M, et al. Could istradefylline be a treatment option for postural abnormalities in mid-stage Parkinson's disease? J Neurol Sci. 2018; 385: 131-133, doi: 10.1016/j. jns.2017.12.027, indexed in Pubmed: 29406892.

7. Kataoka $H$, Sugie $K$. Does istradefylline really have a dystonic mechanism? J Neurol Sci. 2018; 388: 233-234, doi: 10.1016/j. jns.2018.03.020, indexed in Pubmed: 29559176.

8. Margraf NG, Wrede A, Deuschl G, et al. Pathophysiological Concepts and Treatment of Camptocormia. J Parkinsons Dis. 2016; 6(3): 485501, doi: 10.3233/JPD-160836, indexed in Pubmed: 27314757.

9. Suzuki M, Hirai T, Ito Y, et al. Pramipexole-induced antecollis in Parkinson's disease. J Neurol Sci. 2008; 264(1-2): 195-197, doi: 10.1016/j.jns.2007.08.008, indexed in Pubmed: 17826796.

10. Taguchi Y, Takashima S, Tanaka K. Pramipexole-induced dropped head syndrome in Parkinson's disease. Intern Med. 2008; 47(22): 2011-2012, doi: 10.2169/internalmedicine.47.1579, indexed in Pubmed: 19015619.

11. Cannas A, Solla P, Floris G, et al. Reversible Pisa syndrome in patients with Parkinson's disease on dopaminergic therapy. J Neurol. 2009; 256(3): 390-395, doi: 10.1007/s00415-009-0072-6, indexed in Pubmed: 19319462. 
12. Uzawa A, Mori M, Kojima S, et al. Dopamine agonist-induced antecollis in Parkinson's disease. Mov Disord. 2009; 24(16): 2408-2411, doi: 10.1002/mds.22779, indexed in Pubmed: 19890970.

13. Nakayama Y, Miwa H. Drug-induced camptocormia: a lesson regarding vascular Parkinsonism. Intern Med. 2012; 51(19): 2843-2844, doi: $10.2169 /$ internalmedicine.51.8469, indexed in Pubmed: 23037490.

14. Yoritaka A, Shimo Y, Takanashi M, et al. Motor and non-motor symptoms of 1453 patients with Parkinson's disease: prevalence and risks. Parkinsonism Relat Disord. 2013; 19(8): 725-731, doi: 10.1016/j. parkreldis.2013.04.001, indexed in Pubmed: 23639756.

15. Galati S, Möller JC, Städler C. Ropinirole-induced Pisa syndrome in Parkinson disease. Clin Neuropharmacol. 2014; 37(2): 58-59, doi: 10.1097/WNF.0000000000000022, indexed in Pubmed: 24614668.

16. Bloch F, Houeto JL, Tezenas du Montcel S, et al. Parkinson's disease with camptocormia. J Neurol Neurosurg Psychiatry. 2006; 77(11): 1223-1228, doi: 10.1136/jnnp.2006.087908, indexed in Pubmed: 16754693.

17. Wrede A, Margraf NG, Goebel HH, et al. Myofibrillar disorganization characterizes myopathy of camptocormia in Parkinson's disease. Acta Neuropathol. 2012; 123(3): 419-432, doi: 10.1007/s00401-0110927-7, indexed in Pubmed: 22160321.

18. Napolitano F, Pasqualetti M, Usiello A, et al. Dopamine D2 receptor dysfunction is rescued by adenosine A2A receptor antagonism in a model of DYT1 dystonia. Neurobiol Dis. 2010; 38(3): 434-445, doi: 10.1016/j.nbd.2010.03.003, indexed in Pubmed: 20227500.

19. Takakusaki K, Kohyama J, Matsuyama K, et al. Medullary reticulospinal tract mediating the generalized motor inhibition in cats: parallel inhibitory mechanisms acting on motoneurons and on interneuronal transmission in reflex pathways. Neuroscience. 2001; 103(2): 511-527, doi: 10.1016/s0306-4522(00)00586-8, indexed in Pubmed: 11246165.

20. Takakusaki K, Saitoh K, Harada $\mathrm{H}$, et al. Role of basal ganglia-brainstem pathways in the control of motor behaviors. Neurosci Res. 2004; 50(2): 137-151, doi: 10.1016/j.neures.2004.06.015, indexed in Pubmed: 15380321.

21. Lazarus M, Shen HY, Cherasse $Y$, et al. Arousal effect of caffeine depends on adenosine A2A receptors in the shell of the nucleus accumbens. J Neurosci. 2011; 31(27): 10067-10075, doi: 10.1523/ JNEUROSCI.6730-10.2011, indexed in Pubmed: 21734299.
22. Furusawa $\mathrm{Y}$, Mukai $\mathrm{Y}$, Kawazoe T, et al. Long-term effect of repeated lidocaine injections into the external oblique for upper camptocormia in Parkinson's disease. Parkinsonism Relat Disord. 2013; 19(3): 350-354, doi: 10.1016/j.parkreldis.2012.09.008, indexed in Pubmed: 23043967.

23. Kataoka $\mathrm{H}$, Ueno $\mathrm{S}$. Can postural abnormality really respond to levodopa in Parkinson's disease? J Neurol Sci. 2017; 377: 179-184, doi: 10.1016/j.jns.2017.04.025, indexed in Pubmed: 28477691.

24. Chen W, Wang $\mathrm{H}$, Wei $\mathrm{H}$, et al. Istradefylline, an adenosine $\mathrm{A} \square \mathrm{A}$ receptor antagonist, for patients with Parkinson's Disease: a meta-analysis. J Neurol Sci. 2013; 324(1-2): 21-28, doi: 10.1016/j. jns.2012.08.030, indexed in Pubmed: 23085003.

25. Sako W, Murakami N, Motohama K, et al. The effect of istradefylline for Parkinson's disease: A meta-analysis. Sci Rep. 2017; 7(1): 18018, doi: 10.1038/s41598-017-18339-1, indexed in Pubmed: 29269791.

26. Tao Y, Liang G. Efficacy of adenosine A2A receptor antagonist istradefylline as augmentation for Parkinson's disease: a meta-analysis of randomized controlled trials. Cell Biochem Biophys. 2015; 71(1): 57-62, doi: 10.1007/s12013-014-0162-7, indexed in Pubmed: 25096504.

27. Takahashi M, Fujita M, Asai N, et al. Safety and effectiveness of istradefylline in patients with Parkinson's disease: interim analysis of a post-marketing surveillance study in Japan. Expert Opin Pharmacother. 2018; 19(15): 1635-1642, doi: 10.1080/14656566.2018.1518433, indexed in Pubmed: 30281377.

28. Fernandez HH, Greeley DR, Zweig RM, et al. 6002-US-051 Study Group. Istradefylline as monotherapy for Parkinson disease: results of the 6002-US-051 trial. Parkinsonism Relat Disord. 2010; 16(1): 1620, doi: 10.1016/j.parkreldis.2009.06.008, indexed in Pubmed: 19616987.

29. Pourcher E, Fernandez HH, Stacy M, et al. Istradefylline for Parkinson's disease patients experiencing motor fluctuations: results of the KW6002-US-018 study. Parkinsonism Relat Disord. 2012; 18(2): 178184, doi: 10.1016/j.parkreldis.2011.09.023, indexed in Pubmed: 22000279.

30. Torti M, Vacca L, Stocchi F. Istradefylline for the treatment of Parkinson's disease: is it a promising strategy? Expert Opin Pharmacother. 2018; 19(16): 1821-1828, doi: 10.1080/14656566.2018.1524876, indexed in Pubmed: 30232916. 